Research Paper

\title{
MicroRNA 21 Inhibits Leit Ventricular Remodeling in the Early Phase of Rat Model with Ischemia-reperiusion Injury by Suppressing Cell Apoptosis
}

\author{
Yanjun Qin1, Yueqing Yu², Hua Dong ${ }^{1}$, Xiaohua Bian1, Xuan Guo1, Shimin Dong1 ${ }^{凶}$ \\ 1. Department of Emergency, the Third Hospital of Hebei Medical University, Shijiazhuang 050051, China; \\ 2. Department of Clinical Laboratory, the General Hospital of Hebei Province, Shijiazhuang 050051, China.
}

$\triangle$ Corresponding author: Shimin Dong, Department of Emergency, the third hospital of Hebei Medical University, Shijiazhuang 050051, China. Tel: 0086-311-88602230; E-mail: lvguangw@hebmu.edu.cn.

( ) Ivyspring International Publisher. This is an open-access article distributed under the terms of the Creative Commons License (http://creativecommons.org/ licenses/by-nc-nd/3.0/). Reproduction is permitted for personal, noncommercial use, provided that the article is in whole, unmodified, and properly cited.

Received: 2012.04.23; Accepted: 2012.07.11; Published: 2012.07.21

\begin{abstract}
Objective: To determine the role of microRNA 2 I (miR-2I) on left ventricular remodeling of rat heart with ischemia-reperfusion (I/R) injury and to investigate the underlying mechanism of miR-2I mediated myocardium protection.

Methods: Rats were randomly divided into three groups: an I/R model group with Ad-GFP (Ad-GFP group), an I/R model group with Ad-miR-2I (Ad-miR-2I group) and a sham-surgery group. Changes in hemodynamic parameters were recorded at I week after I/R. Histological diagnosis was achieved by hematoxylin and eosin (H\&E). Left ventricular (LV) dimensions, myocardial infarct size, LV/BW, collagen type I, type III and PCNA positive cells were measured. Primary cultures of neonatal rat cardiac ventricular myocytes were performed and cell ischemic injury was induced by hypoxia in a serum- and glucose-free medium, and reoxygenation $(H / R)$.MiR-2I inhibitor and pre-miR-2I were respectively added to the culture medium for the miR-2I knockdown and for the miR-2I up-regulation. QRT-PCR was used to determine the miR-2I levels in cultured cells. Flow cytometry was performed to examine the cell apoptosis.

Results: In the Ad-miR-2I group, LV dimensions, myocardial infarct size, LV/BW, collagen type I , type III and PCNA positive cells all significantly decreased combared with the Ad-GFP groud. At I week after I/R. the Ad-miR-2 I significantly improved LVSP. LV $+\mathrm{db} / \mathrm{d} t$.... $\mathrm{LV}-\mathrm{dp} / \mathrm{d} t_{\min }$, and decreased heart rate (HR) and LVEDP compared with the Ad-GFP group. Compared with the Ad-GFP, the cell apoptotic rate significantly decreased in the Ad-miR-2I group. The miR-2I inhibitor exacerbated cardiac myocyte apoptosis and the pre-miR-2I decreased hypoxia/reoxygenation- induced cardiac myocyte apoptosis.

Conclusions: Ad-miR-2I improves LV remodeling and decreases the apoptosis of myocardial cells, suggesting the possible mechanism by which Ad-miR-2I functions in protecting against I/R injury.
\end{abstract}

Key words: microRNA 21; ischemia-reperfusion; ventricular remodeling; hemodynamic; collagen; apoptosis; rat.

\section{Introduction}

Acute myocardial infarction (MI) due to coronary artery occlusion represents a major cause of morbidity and mortality in humans [1]. Heart failure is an unfavorable consequence of myocardial remod- eling after AMI [2]. Cardiac structural remodeling is associated with myocardium repair reactions, followed by scar formation in the infarcted area as well as changes in the noninfarcted myocardium, includ- 
ing interstitial fibrosis and collagen remodeling [3]. Myocardial fibrosis, a characteristic of all forms of cardiac pathology, leads to mechanical stiffness, which contributes to ventricular contractile dysfunction [4].

MicroRNAs (miRNAs) are endogenous, noncoding, single-stranded RNAs of $\sim 22$ nucleotides and constitute a novel class of gene regulators [5-7]. Currently, about 700 miRNAs have been cloned and sequenced in humans, and the estimated number of miRNA genes is as high as 1,000 in the human genome $[8,9]$. Functionally, an individual miRNA is as important as a transcription factor because it is able to regulate the expression of its multiple target genes. As a group, miRNAs are estimated to regulate over $30 \%$ of the genes in a cell [10]. It is thus not surprising that miRNAs are involved in the regulation of almost all the major cellular functions, such as apoptosis and necrosis, which are two key cellular events in cardiac remodeling after AMI.

Recent studies have identified signature expression patterns of miRNAs associated with pathological cardiac hypertrophy, heart failure, and myocardial infarction in humans and mouse models of heart disease [11]. MicroRNA-21(miR-21) that is consistently induced by cardiac stress, appears to function as a regulator of cardiac growth and fetal gene activation in primary cardiomyocytes in vitro $[12,13]$. Pre-injected with heat-shock-induced miRNAs including miR-21 in the mouse hearts reduces myocardial infarct size after I/R injury [14]. We have also confirmed the aberrantly expressed miR-21 has a protective effect on myocardial infarction and protective effect on $I / R$ by reducing cardiac cell apoptosis via its target gene PDCD4 and AP-1 pathway $[15,16]$.

A subset of miRNAs is enriched in cardiac fibroblasts compared to cardiomyocytes, including miR-21 and members of the miR-29 family [17]. It is well established that miR-21 functions as an oncogene and has a role in tumorigenesis by promoting cell proliferation [18]. Thum $\mathrm{T}$ et al [17] showed in vivo silencing of miR-21 by a specific antagomir in a mouse pressure-overload-induced disease model reduces cardiac ERK-MAP kinase activity, inhibits interstitial fibrosis and attenuates cardiac dysfunction. In Eva van Rooij's study down-regulation of miR-29 with anti-miRs in vitro and in vivo induces the expression of collagens, whereas over-expression of miR-29 in fibroblasts reduces collagen expression [18]. Thum et al. reported that knockdown of miR-21 through systemic delivery of cholesterol-modified antisense oligonucleotides (antagomirs) prevented cardiac hypertrophy and fibrosis in response to pressure overload induced by transaortic constriction (TAC). In the cur- rent study, the treatment effects of miR-21 on myocardium remodeling, including cardiac hemodynamic measurement and collagen remodeling and its mechanism in an in vivo rat model with $I / R$ injury were investigated.

\section{Materials and methods}

\section{Establishment of the rat I/R model}

$\mathrm{I} / \mathrm{R}$ was induced in 10-week-old male Sprague-Dawley rat hearts (weighting 250-300 g) as described in our previous study [15]. In brief, the rats were anesthetized with ketamine $(80 \mathrm{mg} / \mathrm{kg}$ i.p.) and xylazine $(5 \mathrm{mg} / \mathrm{kg}$ i.p.). Under sterile conditions, the heart was exposed through a left thoracotomy in the fourth intercostal space. I/R was induced by $60-\mathrm{min}$ occlusion of the left anterior descending coronary artery (LAD) with a silk suture, followed by one-week reperfusion of LAD. Sham-operated rats served as controls. Sham operation involved an identical procedure, except the suture was passed around the vessel without left anterior descending coronary artery occlusion. All protocols were approved by the Animal Ethics Committee of Hebei Medical University.

\section{Construction of the adenovirus expressing miR-2I, or control adenovirus expressing GFP}

The adenovirus expressing miR-21 (Ad-miR-21), or control adenovirus expressing GFP (Ad-GFP) were generated using the Adeno-XTM Expression Systems 2 kit (Clontech) according to the manufacturer's protocols. These adenoviruses were purified by cesium chloride gradient ultracentrifugation and titrated using a standard plaque assay.

\section{Adenovirus-mediated miR-2I gene transfer in vivo}

Ad-miR-21 or Ad-GFP were delivered into the rat hearts 3 days before I/R. Briefly, the rats were anesthetized with ketamine $(80 \mathrm{mg} / \mathrm{kg}$ i.p.) and xylazine (5 mg/kg i.p.). The pericardium was opened via the third intercostal space. The aorta and pulmonary artery were identified. A 23-gauge catheter containing $200 \mu \mathrm{l}$ of adenovirus was advanced from the apex of the left ventricle to the aortic root. The aorta and pulmonary arteries were clamped distal to the site of the catheter and the solution was injected. The clamp was maintained for $10 \mathrm{~s}$ when the heart pumped against a close system (isovolumically). This procedure allows the solution that contains the adenovirus to circulate down the coronary arteries and perfuse the heart. After $10 \mathrm{~s}$, clamps on the aorta and pulmonary artery were released and the chest was 
closed.

\section{Hemodynamic examination}

Rats were anesthetized with ketamine (80 $\mathrm{mg} / \mathrm{kg}$ i.p.) and xylazine (5 $\mathrm{mg} / \mathrm{kg}$ i.p.). Catheters were inserted into the left ventricle through the right common carotid artery. Changes in hemodynamic parameters, including the heart rate (HR), the left ventricular end-diastolic pressure (LVEDP), the left ventricular systolic pressure (LVSP), the maximal rate of rise in blood pressure in the ventricular chamber $(+\mathrm{dP} / \mathrm{dt}$ max $)$ and the maximal rate of decline in blood pressure in the ventricular chamber (-dP/dtmax) were measured and recorded by an 8-channel polygraph system (RM-6000).

\section{Cardiac histology}

Detailed cardiac histology including immunohistochemistry was performed on the Ad-GFP and Ad-miR-21 rats from the first experiment. After hemodynamic measurements, the hearts were arrested in diastole with $15 \%$ potassium chloride and fixed by immersion in $10 \%$ buffered formalin for $24-48 \mathrm{~h}$. Three transverse slices from the base, mid-region and apex were embedded in paraffin. Then each slice was sectioned ( $6 \mu \mathrm{m}$ thick) for histology and immunohistochemistry. Sections were stained with hematoxylin and eosin.

\section{Measurement of infarct size and determina- tion of infarcted, border, and noninfarcted areas}

At one week after I/ $R$, the rats were anesthetized and $6 \mathrm{ml}$ of $1 \%$ Evans blue dye was injected into the vena cava to delineate the noninfarcted portion of the heart. The myocardial ischemic area at risk was identified as the region lacking blue staining. The ventricles of the hearts were sliced transversely into 2-mm thick slices. The slices were incubated in $1 \%$ triphenyltetrazolium chloride (TTC) at $37^{\circ} \mathrm{C}$ for $30 \mathrm{~min}$ to identify the noninfarcted and infarcted areas. Infarct size was expressed as a percentage of the ischemic area at risk. In myocardial slices, noninfarcted area was defined as the Evans blue-stained area. The infarcted area was displayed as the TTC unstained area. The border area was identified as Evans blue unstained and TTC-stained areas.

\section{Immunohistochemistry}

The myocardial slices were fixed in $4 \%$ paraformaldehyde in $0.1 \mathrm{~mol} / \mathrm{L}$ phosphate buffer and embedded in paraffin, $4-\mu \mathrm{m}$ cross sections were cut from each slice. The sections were deparaffinized and incubated for 10 minutes with $3 \% \mathrm{H}_{2} \mathrm{O}_{2}$ in methanol at room temperature, endogenous peroxidase activity was quenched and sections were washed in phosphate-buffered saline (PBS). They were put into waterbath to repair antigens for $20 \mathrm{~min}$ at $98^{\circ} \mathrm{C}$ with citric acid buffer, then taken out and cooled at room temperature. Add 10\% normal goat serum blocking solution to each slide. The sections were incubated with diluted primary antibodies against collagen I, collagen III, PCNA and incubated at $4^{\circ} \mathrm{C}$ overnight. The sections were then washed with PBS containing 1\% bovine serum albumin three times before incubation with biotinylated secondary antibody (Santa Cruz Biotechnology, USA) for 30 minutes at $37^{\circ} \mathrm{C}$ and labeled with peroxidase avidin using diaminobenzidine as the chromogen. The sections were counterstained with hematoxylin and then blocked. A rabbit anti-rat antibody and a rat PCNA monoclonal antibody were used as primary antibodies. Secondary antibodies were biotinylated using goat anti-rabbit IgG. A light microscope was used for qualitative analysis of the section $(\times 400)$. Ten sections were obtained from each group and the expression of collagen I, collagen III and the number of cells with PCNA positive were assessed. The area of collagen I or III in the border-zone area was counted using light microscopy by an investigator blind to the identity of the samples in 8 randomly selected areas and subsequently averaged. The ratio of collagen I / III was calculated.

\section{Measurement of dimensions of the Left ven- tricles (LV) and LV relative weight}

One week after $I / R$, the anesthetized rat hearts were arrested in diastole by an intravenous injection of saturated potassium chloride solution via the left jugular vein. The heart was excised and LV was separated and weighed. The dimensions of the LV were measured in transverse slices at the level of papillary muscle as described $[19,20]$. LV relative weight was the ratio $\mathrm{LV} / \mathrm{BW}$.

\section{Cardiacmyocyte culture and cell ischemia in- jury model}

Primary cultures of neonatal rat cardiac ventricular myocytes were performed as described previously [15, 21]. In brief, hearts from 1-2-day-old Sprague-Dawley rats were placed in ice-cold $1 \times$ phosphate-buffered saline solution. After repeated rinsing, the atria were cut off, and the ventricles were minced with scissors. The minced tissue and ventricular cells were dispersed by digestion with collagenase type IV $(0.45 \mathrm{mg} / \mathrm{ml}), 0.1 \%$ trypsin, and $15 \mu \mathrm{g} / \mathrm{ml}$ DNase I. Cardiomyocytes $\left(0.33 \times 10^{6}\right.$ cells $\left./ \mathrm{ml}\right)$ were cultured in cardiac myocyte culture medium containing Dulbecco's modified Eagle's medium/F-12 supplemented 
with $5 \%$ horse serum, $4 \mu \mathrm{g} / \mathrm{ml}$ of transferrin, 0.7 $\mathrm{ng} / \mathrm{ml}$ of sodiumselenite, $2 \mathrm{~g} /$ liter of bovine serum albumin, $3 \mathrm{mmol} /$ liter of pyruvic acid, $15 \mathrm{mmol} /$ liter of HEPES, $100 \mu \mathrm{mol} /$ liter of ascorbic acid, $100 \mu \mathrm{g} / \mathrm{ml}$ of ampicillin, $5 \mu \mathrm{g} / \mathrm{ml}$ of linoleic acid, $1 \%$ penicillin, $1 \%$ streptomycin, and $100 \mu \mathrm{mol} / \mathrm{liter}$ 5-bromo-2'-deoxyuridine and seeded into six-well plates.

Cell ischemic injury was induced by hypoxia in a serum- and glucose-free medium, and reoxygenation $(\mathrm{H} / \mathrm{R})$. Hypoxia was achieved by placing the cells in a hypoxia chamber filled with $5 \% \mathrm{CO}_{2}$ and $95 \% \mathrm{~N}_{2}$ at 37 ${ }^{\circ} \mathrm{C}$ for $4 \mathrm{~h}$. Following hypoxia exposure, the cells were reoxygenated with $5 \% \mathrm{CO}_{2}$ and $95 \% \mathrm{O}_{2}$ for $3 \mathrm{~h}$ in Dulbecco's modified Eagle's medium containing 5\% serum and normal glucose.

\section{Oligo transfection, miR-2I knockdown and miR-2I overexpression in cultured cardiac myocytes}

Oligo transfection was performed according to an established protocol [22, 23]. For the miR-21 knockdown, miR-21 inhibitor (LNA-anti-miR-21) (Exiqon, Inc.) was added to the culture medium at a final oligonucleotide concentration of $30 \mathrm{nM}$. For miR-21 up-regulation, pre-miR-21 (Ambion, Inc.) was added directly to the complexes at a final oligonucleotide concentration of $30 \mathrm{nM}$. The transfection medium was replaced $4 \mathrm{~h}$ post-transfection by regular culture medium. Vehicle control, oligo controls for LNA-anti-miR-21 and pre-miR-21 (Ambion, Inc.), and adenovirus control (Ad-GFP) were applied.

\section{Quantitative reverse transcriptase-PCR (qRT-PCR)}

qRT-PCR was used to determine the miR-21 levels in cultured cells. qRT-PCR was performed on cDNA generated from $50 \mathrm{ng}$ of total RNA using the protocol of the mirVana qRT-PCR miRNA Detection Kit (Ambion, Inc). Amplification and detection of specific products were performed with a Roche Lightcycler 480 Detection System. As an internal control, U6 was used for template normalization. The primers used were provided by Ambion, Inc. Fluorescent signals were normalized to an internal reference, and the threshold cycle $(\mathrm{Ct})$ was set within the exponential phase of the PCR. The relative gene expression was calculated by comparing cycle times for each target PCR. The target PCR Ct values were normalized by subtracting the $\mathrm{U} 6 \mathrm{Ct}$ value, which provided the $\Delta \mathrm{Ct}$ value. The relative expression level between treatments was then calculated using the following equation: relative gene expression $=2^{-(\Delta}$ $\mathrm{Ct}$,sample- $\Delta \mathrm{Ct}$, control).

\section{Flow cytometry analysis}

Cardiac myocyte apoptosis in border areas and in cultured cells with ischemia injury was measured by flow cytometry. Annexin V was labeled as described previously[24, 25]. The infarcted, border, and noninfarcted areas were identified as described as above. Briefly, isolate cardiomyocytes for flow cytometry, then detect cell apoptosis with Annexin V-FITC/PI Apoptosis Detection Kit (Calbiochem, Merck) according to the manufacturer's protocol. Cells were harvested and adjusted to a confluency of $0.5 \times 10^{6}$ to $1 \times 10^{6}$ cells $/ \mathrm{mL}$. Subsequently, cells were fixed with $70 \%$ pre-chilled alcohol and stained with propidium iodide (PI). PI-labeled cells were analyzed using flow cytometry.

\section{Statistical analysis}

Statistical analyses were performed using SPSS 10.0 software. Data were presented as mean \pm standard deviation (SD). The difference between two groups was evaluated by un-paired $t$ test. The differences among different groups were determined by Kruskal-Wallis test. A probability $(P)$ - value of $<0.05$ was considered statistically significant.

\section{Results}

\section{Hemodynamic examination}

Hemodynamic index was recorded at 1 week after I/R. In this experiment, as shown in Fig.1, Ad-miR-21 significantly improved LVSP, $\mathrm{LV}+\mathrm{d} p / \mathrm{d} t_{\max }, \mathrm{LV}-\mathrm{d} p / \mathrm{d} t_{\min }$, and decreased $\mathrm{HR}$, LVEDP compared with the Ad-GFP group. These observations suggested that Ad-miR-21 improved the heart function in rats with I/R injury.

\section{The effect of adenovirus-mediated miR-2 I gene transfer on LV/BW}

As shown in Fig.2, at 1 week after I/R, Ad-miR-21 significantly decreased the ratio LV/BW compared with the Ad-GFP group.

\section{Measurement of myocardial infarct size}

In our previous study[15], at 3 days after administration, Ad-miR-21 increased miR-21 expression in rat hearts in a dose-dependent manner. Based on the dose response of Ad-miR-21, we injected $8 \times 10^{8}$ $\mathrm{pfu} /$ rat into the animals using our delivery method at 3 days before I/R. As shown in Fig. 3A, compared with the control adenovirus (Ad-GFP)-treated rats, Ad-miR-21 reduced myocardial infarct size by $15.8 \pm$ $0.90 \%$ at 1 week after I/R. Representative TTC-stained heart slices from the rats treated with Ad-miR-21 or Ad-GFP are shown in Fig. 3B. 

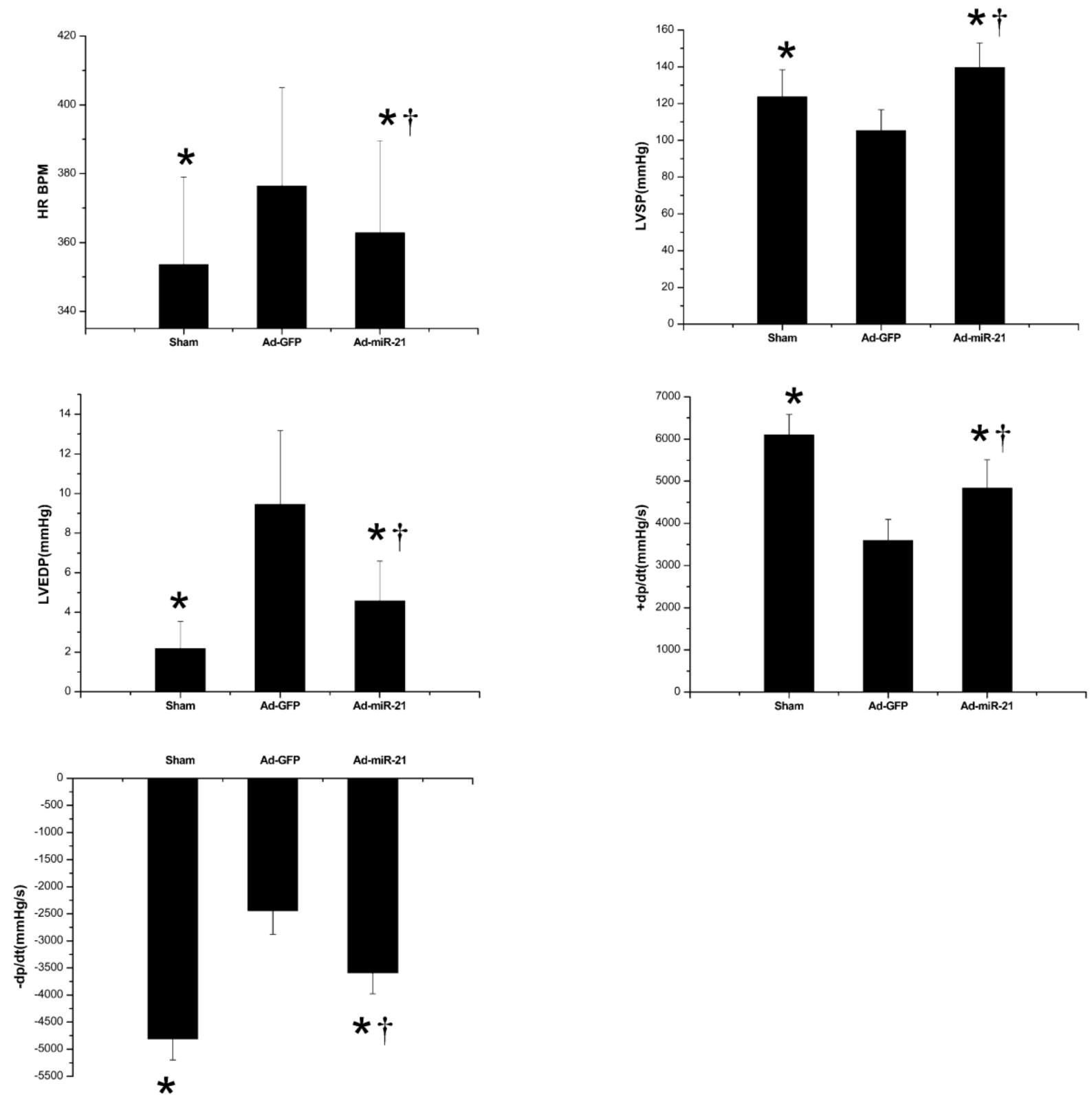

FIGURE I. Cardiac hemodynamics measured at I week after I/R. HR is heart rate. BPM is beats per minute. LVSP is left ventricular systolic pressure. $\mathrm{LV} \pm d p / d t$ are the first derivatives (positive and negative) of LV pressure over time. LVEDP is left ventricular end-diastolic pressure. Results are expressed as mean $\pm S D, n=10$. $* P<0.05$ compared with the Ad-GFP group. $\dagger P<0.05$ compared with the sham group.

FIGURE 2. The ratio LV/BW measured I week after I/R. LV is left ventricular. BW is body weight. Results are expressed as the mean \pm SD $* P<0.05$ compared with the Ad-GFP group. $\dagger P<0.05$ compared with the sham group.






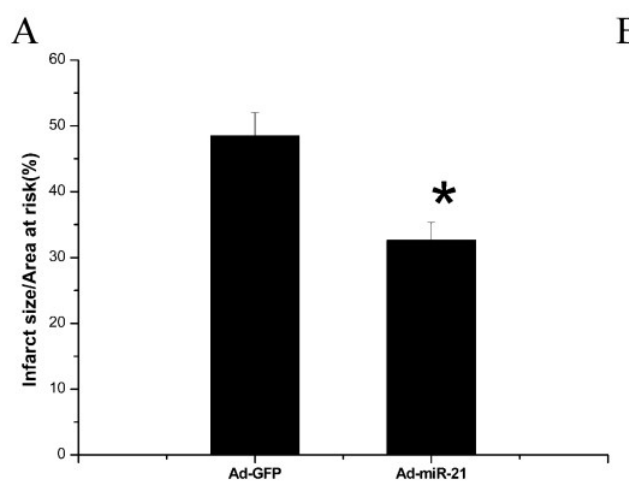

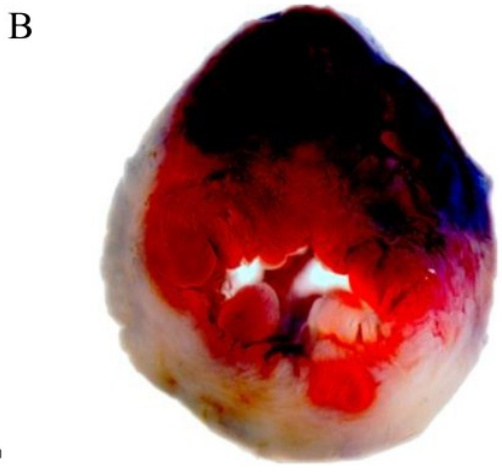

Ad-GFP

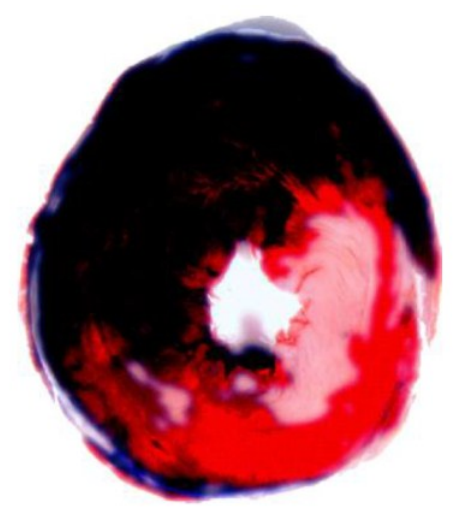

Ad-miR-21

FIGURE 3. The effect of adenovirus-mediated miR-2I gene transfer on myocardial infarct size. A, Ad-miR-2I ( $8 \times 10^{8}$ pfu) reduced infarct size in rat hearts at I week after I/R. Note: Data presented as mean \pm SD, $n=10 ; *, p<0.05$ compared with Ad-GFP control. B, representative TTC-stained heart slices from rats treated with control adenovirus, Ad-GFP, or Ad-miR-2I. The infarcted area in the Ad-GFP-treated image was $48.5 \%$ and the infarcted area in the Ad-miR-2I-treated image was $32.7 \%$.

\section{Content of collagen type I, type III and PCNA positive cells with immunohistochemistry}

The effect of Adenovirus-mediated miR-21 Gene Transfer on collagen type I, type III and PCNA positive cells. As shown in Fig.4 compared with the Ad-GFP, the collagen type I and type III content in both noninfarcted area and infarcted area significantly decreased in the Ad-mirR-21 group. The ratio of collagen type I / III had the same result. Compared with the Ad-GFP group, PCNA positive cells in the border area significantly decreased in the Ad-miR-21 group.

\section{Flow cytometry analysis}

Ad-miR-21 suppressed cell apoptosis in the border area of the rat heart with I/R injury. As shown in Fig. 5B, compared with the Ad-GFP group, the cell apoptotic rate significantly decreased in the Ad-miR-21 group.

\section{The effect of miR-2I on ischemia-related cell injury in vitro}

To determine the potential cellular mechanism behind miR-21-mediated protective effects during myocardial infarction, an ischemia-related cell injury model was applied in which injury to the cultured cells was induced by hypoxia in a serum- and glucose-free medium, followed by reoxygenation in normal culture medium. We found that the miR-21 inhibitor, LNA-anti-miR-21 (30nM), decreased, but
pre-miR-21 increased miR-21 expression in cultured cardiac cells (Fig. 6A). As expected, hypoxia/reoxygenation resulted in an increase in apoptosis. Notably, pre-miR-21 decreased hypox$\mathrm{ia/reoxygenation-induced} \mathrm{cardiacmyocyte} \mathrm{apoptosis}$ (Fig. 6B). In contrast, cardiac myocyte apoptosis was exacerbated after treatment with LNA-anti-miR-21 (Fig.6B).

The reduced myocardial size and heart cell apoptosis should have functional results on LV remodeling such as changes in LV dimensions. To test these results, the infarcted hearts from both the Ad-GFP and Ad-miR-21 pre-treated rats were isolated at 1 week after I/R. As shown in Fig.7, the LV internal diastolic diameter in the Ad-miR-21-treated animals $(7.64 \pm 1.01 \mathrm{~mm})$ was significantly smaller than that in the Ad-GFP-treated animals $(9.27 \pm 1.17 \mathrm{~mm})$.

\section{The effect of adenovirus-mediated miR-2 I gene transfer on LV dimensions}

The reduced myocardial size and heart cell apoptosis should have functional results on LV remodeling such as changes in LV dimensions. To test these results, the infarcted hearts from both the Ad-GFP and Ad-miR-21 pre-treated rats were isolated at 1 week after I/R. As shown in Fig.7, the LV internal diastolic diameter in the Ad-miR-21-treated animals $(7.64 \pm 1.01 \mathrm{~mm})$ was significantly smaller than that in the Ad-GFP-treated animals $(9.27 \pm 1.17 \mathrm{~mm})$. 
A

Sham

type I

ypeIII

PCNA

B

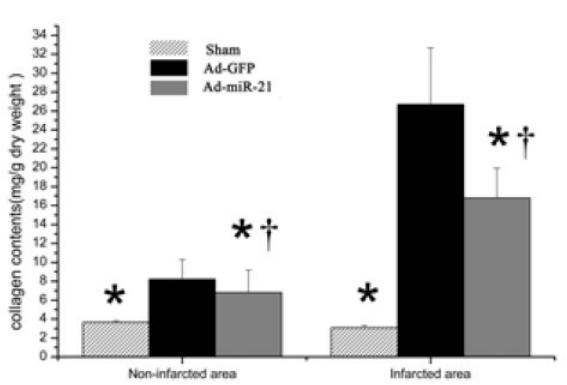

Ad-GFP


C

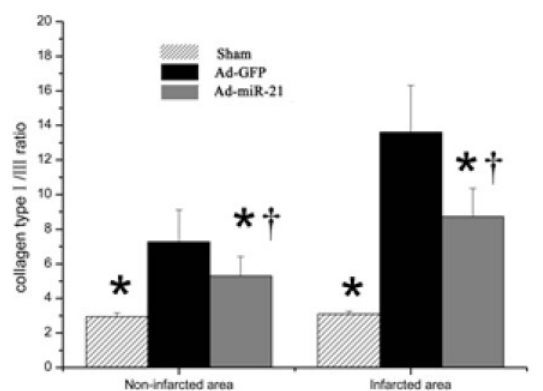

Ad-miR-21
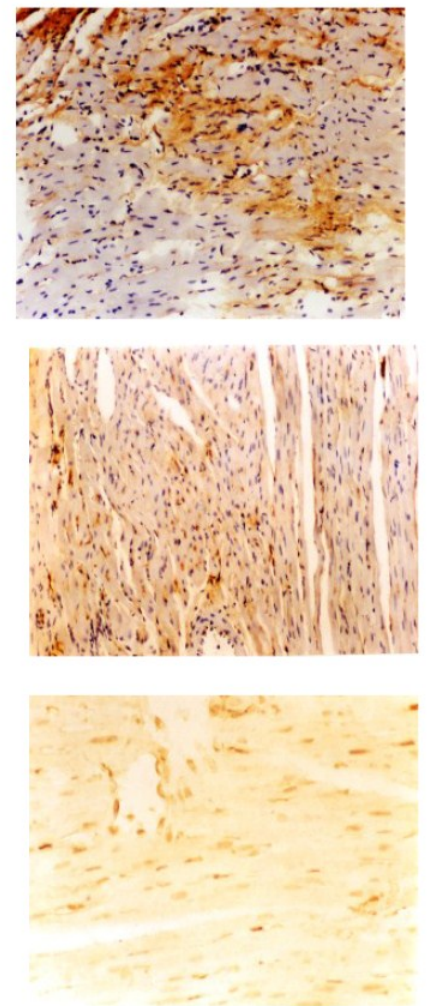

D

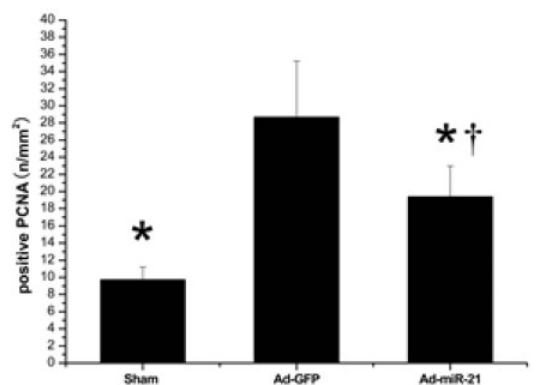

FIGURE 4. A representative collagen type I, type III and PCNA positive cells photomicrographs from the rat heart sections at I week after I/R (immunohistochemistry, original magnification $\times 400$ ). The positive expression colored brown-yellow. B collagen content in noninfarcted area and infarcted area. Note: Data presented as mean $\pm S D, n=6$. C collagen type I / III ratio in noninfarcted area and infarcted area. Note: Data presented as mean $\pm S D, n=6$. D the PCNA positive cells in the border area. $* p<0.05$ compared with the Ad-GFP control. $\uparrow P<0.05$ compared with the sham group. 


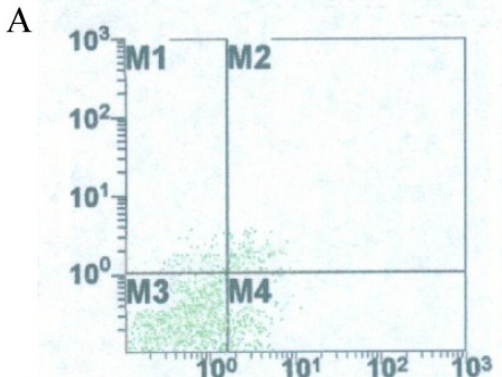

Sham

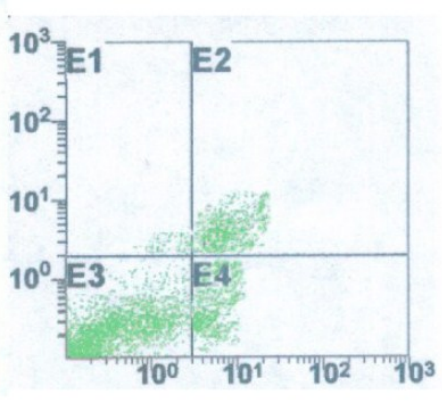

Ad-GFP

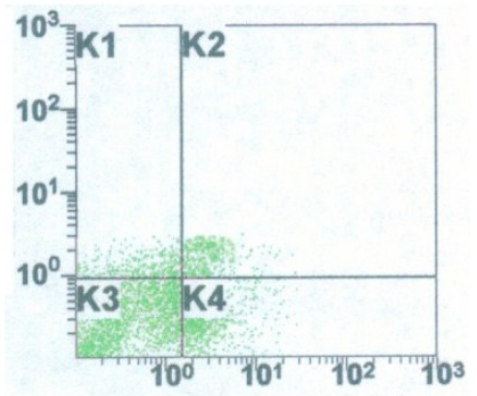

Ad-miR-21

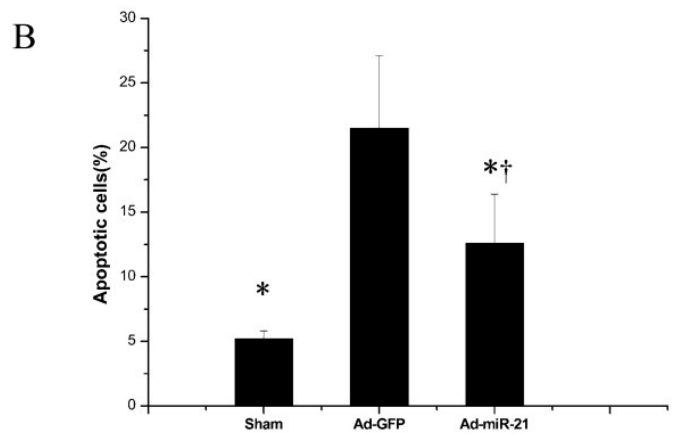

FIGURE 5. The effect of miR-2I overexpression on cardiac cell apoptosis in the border area of the rat heart. A, representative apoptotic cells by flow cytometry I week after I/R. B, quantitative analysis of the apoptotic cells in heart sections. Note: Data presented as mean \pm $\mathrm{SD}, \mathrm{n}=6, *, p<0.05$ compared with the Ad-GFP group. $\dagger P<0.05$ compared with the sham group.
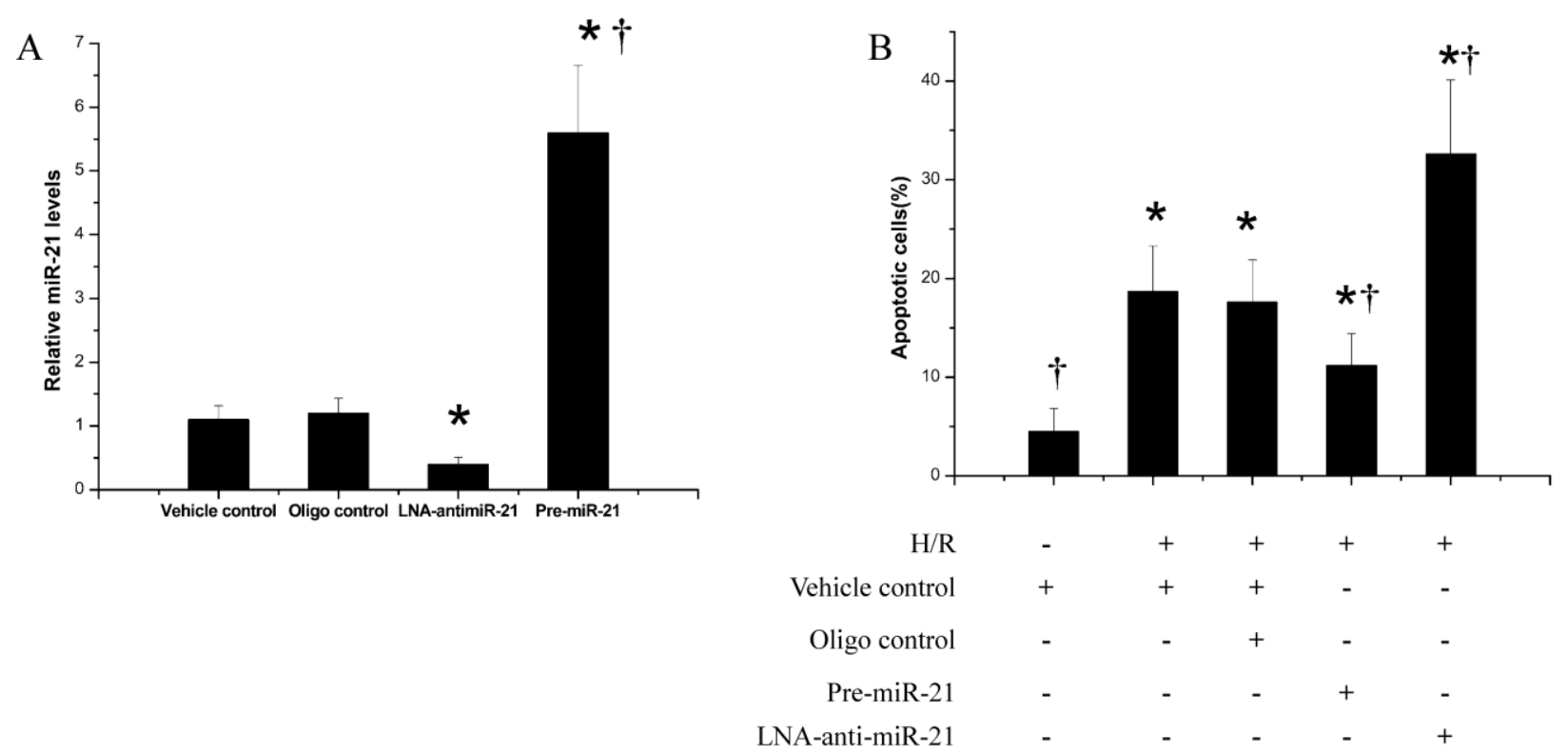

FIGURE 6. The effect of miR-2I on ischemia-related cell injury in vitro. Cultured cardiac cell injury was induced by hypoxia for $4 \mathrm{~h}$ in a serum- and glucose-free medium followed by reoxygenation $(\mathrm{H} / \mathrm{R})$ for $3 \mathrm{~h}$ innormal culture medium. Cell apoptosis was determined by flow cytometry. A, miR-2I inhibitor, LNA-anti-miR-2I (30 nM) decreased, but pre-miR-2I (30 nM) increased miR-2I expression in cultured cardiac cells. $n=5, *, p<0.05$ compared with the vehicle control. $\dagger, p<0.05$ compared with the LNA-anti-miR-2I group. B, hypoxia/reoxygenation resulted in an increase in apoptosis. Pre-miR-2I decreased the cardiac myocyte apoptosis. In contrast, cardiac myocyte apoptosis was exacerbated after treatment with LNA-anti-miR-2I. $n=5, *, p<0.05$ compared with the noninjured (without $\mathrm{H} / \mathrm{R}$ ) control. $\dagger, p<0.05$ compared with $\mathrm{H} / \mathrm{R}$ treated with the vehicle control. 


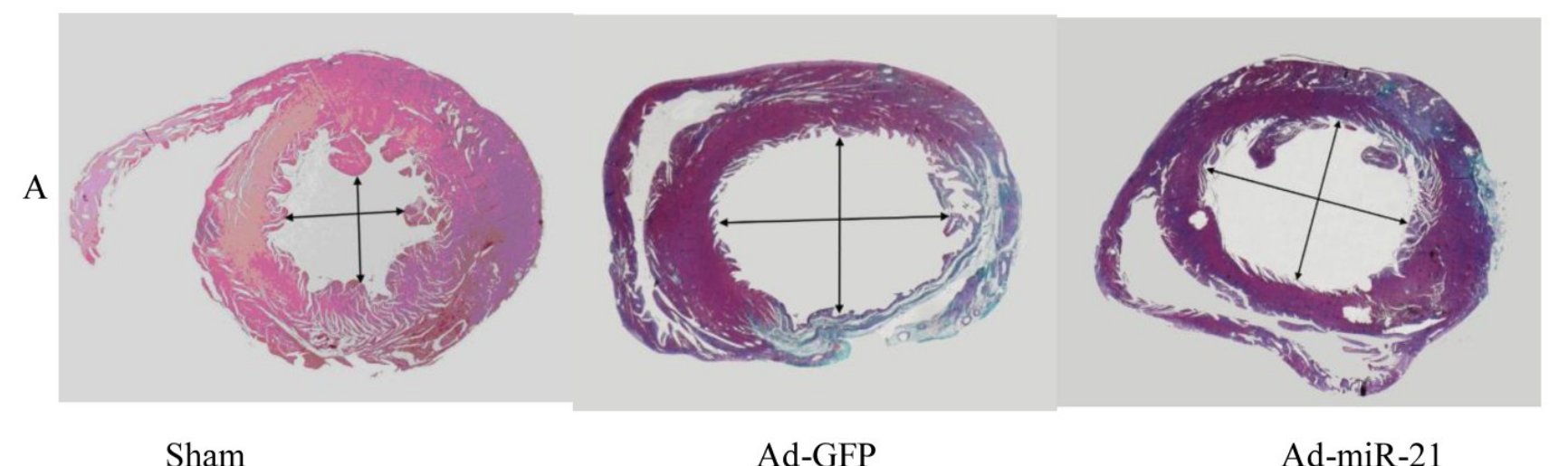

B



FIGURE 7. The effect of adenovirus-mediated miR-2I gene transfer on LV dimensions. A, representative heart sections from sham-opened, Ad-GFP, or Ad-miR-2 I-treated rats at I week after I/R. B, Ad-miR-2I ( $\left.8 \times 10^{8} \mathrm{pfu}\right)$ reduced internal diastolic diameter of left ventricles in rat hearts at I week after I/R. Note: Data presented as mean $\pm \mathrm{SD}, \mathrm{n}=8 ; *, P<0.05$ compared with Ad-GFP control. $\dagger P<0.05$ compared with the sham group.

\section{Discussion}

Our previous study [15] showed that in the rat acute myocardial infarction model, ad-miR-21 increases miR-21 expression in rat myocardium, which reduces myocardial infarct size, left ventricle dimension and cell apoptosis in both border and infarcted areas. In recent years, studies about the effect of miR-21 on ventricular remodeling and myocardial fibrosis do not get consistent results. Thum et al [26] reported that in vivo silencing of miR-21 by a specific antagomir in a mouse pressure-overload-induced disease model inhibits interstitial fibrosis and attenuates cardiac dysfunction. Patrick et al [27] reported that treated with antimiR-21 in pressure overload mice, HW/BW ratio and cardiac function by echocardiography have no significant difference compared with the untreated animals. In MI mice model induced by LAD occlusion, the extent of scar formation and remodeling is indistinguishable between mice of the two genotypes, and quantification of infarct size revealed no significant differences between $\mathrm{WT}$ and miR21-/- mice. These differences may be explained by compensatory mechanisms activated in the per- sistent absence of miR-21 [27].

It is well established that elevated HR increases myocardial oxygen consumption in normal myocardium. HR reduction has been demonstrated to ameliorate atherosclerosis and vascular inflammation, with an improvement in cardiovascular outcome [28]. This study showed that in rat myocardial with I/R injury, ad-miR-21 improved LVSP, decreased HR and LVEDP, improved $\pm \mathrm{dp} / \mathrm{dt}$, and decreased left ventricular relative mass at 1 week after I/R, which improved left ventricular function and hemodynamic index.

Ventricular remodeling by ischemic myocardial injury is an important cause affecting heart function and hemodynamic, which is mainly affected by myocardial infarction size and myocardial collagen remodeling in noninfarcted region. Once the ischemic myocardial injury occurs, the myocardial infarction size is mainly affected by the myocardial cell apoptosis in the border area. Overexpression of miR-21 reduced cell apoptosis in the border areas at early stage of AMI and reduced the myocardial infarct size in the rat heart [15]. Apoptosis is a major cause for cardiac 
infarction following ischaemic preconditioning $(\mathrm{I} / \mathrm{P})$ $[29,30]$. The inhibition of miR-21 has been shown to suppress cell growth by increasing apoptosis and decreasing cell proliferation [31].In contrast, knockdown of miR-21 in cultured glioblastoma cells triggers activation of caspases and leads to increased apoptotic cell death [32]. In the present study, cardiomyocyte apoptosis rate decreased and myocardial infarction size reduced in the miR-21 group compared with the control group at 1 week after myocardial I/R injury. The attenuation of myocardial infarction with miR-21 may be related to reduce cell apoptosis. Yin C et al [14] provided evidence for protective effect of miR-21 on cardiac injury by reducing expression of apoptotic genes-Bid and Bcl-10, which can bind to Bcl-2 to promote apoptosis. Our previous study showed that adenoviral transfer of miR-21 in vivo decreases cell apoptosis in the border and infracted areas through its target gene, programmed cell death 4(PDCD4), and activator protein 1 (AP1) pathway [15]. MiR-21 is induced by the AKT pathway and mediates an antiapoptotic effect via suppression of FasL [33]. Conversely, it activates AKT through suppression of phosphatase and tensin homolog (PTEN) through a feed forward loop [33].

Cardiac fibrosis is a remodeling feature in hearts with ischemic heart disease. The fibrillar collagens, type I and type III, are the major components of the cardiac extracellular matrix [34]. Type I collagen mainly provides rigidity, whereas type III collagen contributes to elasticity. Collagen remodeling occurs following $I / R$ cardiac injury. This remodeling is mainly manifested as reparative fibrosis in infarcted area, while as reactive fibrosis in both noninfarcted and border areas. Collagen remodeling in noninfarcted area plays a main role in ventricular remodeling following I/R cardiac injury. Type I collagen increases and type III collagen decreases, which improves myocardial stiffness, decreases myocardial compliance, as a result of cardiac systolic and diastolic function decreased. This study showed that overexpression of miR-21 decreased collagen content, collagen I / III ratio, which suggested it had improved myocardial collagen I, III remodeling. Furthermore, expression of the proliferation marker PCNA in cardiac fibroblasts was increased in Ad-GFP group, whereas PCNA expression significantly reduced in Ad-miR-21 group. The protective effect of miR-21 against I/ $R$ induced heart damages was approved by the animal experiments [14, 35].Yin et al. found that myocardial infarct size was significantly reduced by treatment with heat-shock- or preconditioning-induced miRNAs including miR-21 in an in vitro mouse model. They further demonstrated that up- regulation of heat-shock protein 70 (HSP70) and of the endothelial and inducible nitric oxide synthases might be responsible for these miRNAs-mediated cardiac protection $[14,35]$.

MiR-21 is also enriched in the myofibroblasts and infiltrating cells in the infarct zone relative to the surrounding myocytes. MiR-21 is significantly upregulated in cardiac fibroblasts in the failing heart, where it represses the expression of sprouty homo$\log 1$ (SPRY1), a negative regulator of the extracellular signal-regulated kinase/mitogen-activated protein (ERK-MAP) kinase signaling pathway [26]. Upregulation of miR-21 in response to cardiac stress was shown to enhance ERK-MAP kinase signaling, leading to fibroblast proliferation and fibrosis. PTEN has also been demonstrated to be a direct target of miR-21 in cardiac fibroblasts [36]. Previous reports characterize PTEN as a suppressor of matrix metalloprotease-2 (MMP-2) expression [37]. I/ $\mathrm{R}$ in the heart induced miR-21 in cardiac fibroblasts in the infracted region. Thus, I/R-induced miR-21 limits PTEN function and causes activation of the Akt pathway and increased MMP-2 expression in cardiac fibroblasts. This effect indirectly contributes to the increase in fibrosis seen during cardiac hypertrophy. But a recent report disputes these results. It shows that global genomic ablation of miR-21, or knockdown with locked nucleic acid (LNA)-modified antisense oligonucleotides, has no impact on the development of fulminant cardiac hypertrophy in response to pressure overload, angiotensin II, calcineurin, or infarction [27]. How might these differences be reconciled? It might be explained by different animal models and different observation methods.

All the analyses showed that overexpression of miR-21 following cardiac I/R injury decreased infarcted area and reduced cell apoptosis in border area, which improved myocardial collagen I, III remodeling in noninfarcted area, improved heart function and hemodynamic status, and inhibited left ventricular remodeling.

\section{Abbreviations}

miRNA, microRNA; TTC, triphenyltetrazolium chloride; pfu, plaque-forming unit; AMI, acute myocardial infarction; Ad, adenovirus; GFP, green fluorescent protein; $\mathrm{LV}$, left ventricle; $\mathrm{BW}$, body weight; $\mathrm{I} / \mathrm{R}$, Ischemia-reperfusion; PCNA, proliferating cell nuclear antigen; PDCD4, programmed cell death 4; LVEF: left ventricular ejection fraction; LVEDP: left ventricular end-diastolic pressure; LVSP: left ventricular systolic pressure; $+\mathrm{dP} / \mathrm{dt}_{\max }$ : maximal rate of rise in blood pressure in ventricular chamber; $-\mathrm{dP} / \mathrm{dt}_{\max }$ : maximal rate of declining in blood pressure in ven- 
tricular chamber; PTEN: Phosphatase and tensin homologue; IP: ischaemic preconditioning

\section{Acknowledgment}

The author's research was supported by Nature Science Foundation of China (No. 81070122) and by the Science and Technology Program of Hebei Province, China (No.C2010000575). The authors would like to thank Professor Yunhui Cheng and Chunxiang Zhang (Department of Anesthesiology, RNA and Cardiovascular Research Laboratory, New Jersey Medical School, University of Medicine and Dentistry of New Jersey) for their excellent experimental technique assistance.

\section{Competing Interests}

The authors have declared that no competing interest exists.

\section{References}

[1] Fox CS, Coady S, Sorlie PD, et al. Increasing cardiovascular disease burden due to diabetes mellitus: the Framingham Heart Study. Circulation. 2007;115:1544-50.

[2] Sun Y. Myocardial repair/remodelling following infarction: roles of local factors. Cardiovasc Res. 2009;81:482-90.

[3] Sun Y, Zhang JQ, Zhang J,et al. Cardiac remodeling by fibrous tissue after infarction in rats. J Lab Clin Med. 2000;135:316-23.

[4] Berk BC, Fujiwara K, Lehoux S. ECM remodeling in hypertensive heart disease. J Clin Invest. 2007;117:568-75

[5] Ambros V. MicroRNA pathways in flies and worms: growth, death, fat, stress, and timing. Cell. 2003;113:673-6.

[6] Farh KK, Grimson A, Jan C, et al. The widespread impact of mammalian MicroRNAs on mRNA repression and evolution. Science. 2005;310:1817-21.

[7] Pasquinelli AE, Hunter S, Bracht J. MicroRNAs: a developing story. Curr Opin Genet Dev. 2005;15:200-5

[8] Bentwich I, Avniel A, Karov Y, et al. Identification of hundreds of conserved and nonconserved human microRNAs. Nat Genet. 2005;37:766-70.

[9] Berezikov E, Guryev V, van de Belt J, et al. Phylogenetic shadowing and computational identification of human microRNA genes. Cell. 2005;120:21-4.

[10] Lewis BP, Burge CB, Bartel DP. Conserved seed pairing, often flanked by adenosines, indicates that thousands of human genes are microRNA targets. Cell. 2005;120:15-20.

[11] van Rooij E, Marshall WS, Olson EN. Toward microRNA-based therapeutics for heart disease: the sense in antisense. Circ Res. 2008;103:919-28.

[12] Cheng Y, Ji R, Yue J, et al. MicroRNAs are aberrantly expressed in hypertrophic heart: do they play a role in cardiac hypertrophy? Am J Pathol. 2007;170:1831-40.

[13] Tatsuguchi M, Seok HY, Callis TE, et al. Expression of microRNAs is dynamically regulated during cardiomyocyte hypertrophy. J Mol Cell Cardiol. 2007;42:1137-41.

[14] Yin C, Wang X, Kukreja RC. Endogenous microRNAs induced by heat-shock reduce myocardial infarction following ischemia-reperfusion in mice. FEBS Lett. 2008;582:4137-42.

[15] Dong S, Cheng Y, Yang J, et al. MicroRNA expression signature and the role of microRNA-21 in the early phase of acute myocardial infarction. J Biol Chem. 2009;284:29514-25.

[16] Cheng Y, Zhu P, Yang J, et al. Ischaemic preconditioning-regulated miR-21 protects heart against ischaemia/reperfusion injury via anti-apoptosis through its target PDCD4. Cardiovasc Res. 2010;87:431-9.

[17] Thum T, Catalucci D, Bauersachs J. MicroRNAs: novel regulators in cardiac development and disease. Cardiovasc Res. 2008;79:562-70.

[18] Tong AW, Nemunaitis J. Modulation of miRNA activity in human cancer: a new paradigm for cancer gene therapy? Cancer Gene Ther. 2008:15:341-55.
[19] Kolakowski S, Jr., Berry MF, et al. Placental growth factor provides a novel local angiogenic therapy for ischemic cardiomyopathy. J Card Surg. 2006;21:559-64.

[20] Zheng B, Cao LS, Zeng QT,et al. Inhibition of NOS2 ameliorates cardiac remodeling, improves heart function after myocardial infarction in rats. Basic Res Cardiol. 2004;99:264-71.

[21] Fu JJ, Gao H, Pi RB, et al. An optimized protocol for culture of cardiomyocyte from neonatal rat. Cytotechnology. 2005;49:109-16.

[22] Ji R, Cheng Y, Yue J, et al. MicroRNA expression signature and antisense-mediated depletion reveal an essential role of MicroRNA in vascular neointimal lesion formation. Circ Res. 2007;100:1579-88.

[23] Cheng Y, Liu X, Zhang S,et al. MicroRNA-21 protects against the $\mathrm{H}(2) \mathrm{O}(2)$-induced injury on cardiac myocytes via its target gene PDCD4. J Mol Cell Cardiol. 2009;47:5-14.

[24] Vermes I, Haanen C, Steffens-Nakken H, et al. A novel assay for apoptosis. Flow cytometric detection of phosphatidylserine expression on early apoptotic cells using fluorescein labelled Annexin V. J Immunol Methods. 1995;184:39-51.

[25] Castedo E, Castejon R, Monguio E, et al. Influence of hypothermia on right atrial cardiomyocyte apoptosis in patients undergoing aortic valve replacement. J Cardiothorac Surg. 2007;2:7.

[26] Thum T, Gross C, Fiedler J, et al. MicroRNA-21 contributes to myocardial disease by stimulating MAP kinase signalling in fibroblasts. Nature. 2008;456:980-4.

[27] Patrick DM, Montgomery RL, Qi X, et al. Stress-dependent cardiac remodeling occurs in the absence of microRNA-21 in mice. J Clin Invest. 2010;120:3912-6.

[28] DiFrancesco D. Cardiac pacemaker I(f) current and its inhibition by heart rate-reducing agents. Curr Med Res Opin. 2005;21:1115-22.

[29] Saraste A, Pulkki K, Kallajoki M,et al. Apoptosis in human acute myocardial infarction. Circulation. 1997;95:320-3.

[30] Olivetti G, Abbi R, Quaini F, et al. Apoptosis in the failing human heart. N Engl J Med. 1997;336:1131-41.

[31] Si ML, Zhu S, Wu H,et al. miR-21-mediated tumor growth. Oncogene. 2007;26:2799-803.

[32] Chan JA, Krichevsky AM, Kosik KS. MicroRNA-21 is an antiapoptotic factor in human glioblastoma cells. Cancer Res. 2005;65:6029-33.

[33] Sayed D, He M, Hong C, et al. MicroRNA-21 is a downstream effector of AKT that mediates its antiapoptotic effects via suppression of Fas ligand. J Biol Chem. 2010;285:20281-90.

[34] Weber KT. Cardiac interstitium in health and disease: the fibrillar collagen network. J Am Coll Cardiol. 1989;13:1637-52.

[35] Yin C, Salloum FN, Kukreja RC. A novel role of microRNA in late preconditioning: upregulation of endothelial nitric oxide synthase and heat shock protein 70. Circ Res. 2009;104:572-5.

[36] Roy S, Khanna S, Hussain SR, et al. MicroRNA expression in response to murine myocardial infarction: miR-21 regulates fibroblast metalloprotease-2 via phosphatase and tensin homologue. Cardiovasc Res. 2009;82:21-9.

[37] Park MJ, Kim MS, Park IC, et al. PTEN suppresses hyaluronic acid-induced matrix metalloproteinase-9 expression in U87MG glioblastoma cells through focal adhesion kinase dephosphorylation. Cancer Res. 2002;62:6318-22. 